EPJ Web of Conferences 70, 00036 (2014)

DOI: $10.1051 /$ epjconf/ 20147000036

(C) Owned by the authors, published by EDP Sciences, 2014

\title{
Heavy lon Physics Highlights from ATLAS
}

\author{
Zvi Citron ${ }^{1, a}$ for the ATLAS Collaboration \\ ${ }^{1}$ Weizmann Institute of Physics
}

\begin{abstract}
An overview of the ATLAS results from $\mathrm{Pb}+\mathrm{Pb}$ collisions at $\sqrt{s_{N N}}=2.76 \mathrm{TeV}$ will be presented. The results for hard probes include both single jet and di-jet measurements, $\mathrm{W}$ and $\mathrm{Z}$ bosons, photons, and high $p_{\mathrm{T}}$ charged tracks. Taken together these results provide a compelling picture of the interaction of hard particles in the dense QCD medium. Additionally, ATLAS has measured properties of the bulk particle production including charged particle multiplicity and extensive measurements of the azimuthal particle distributions and correlations. Results shown will be from the $\sim 10$ inverse $\mu \mathrm{b}^{-} 1$ of minimum bias recorded in the 2010 LHC heavy ion run, as well as from $\sim 0.15 \mathrm{nb}^{-1} 1$ sampled in the 2011 LHC heavy ion run.
\end{abstract}

\section{Introduction}

Lead-Lead collisions at $\sqrt{s_{N N}}=2.76 \mathrm{TeV}$ in the Large Hadron Collider (LHC) provide the opportunity to study strongly interacting matter at the highest temperatures achieved in the laboratory. The ATLAS experiment has a robust heavy-ion program to take advantage of this opportunity. In the 2010 and 2011 LHC runs, yielding approximately $10 \mu \mathrm{b}^{-} 1$ and $0.15 \mathrm{nb}^{-1}$, respectively, the ATLAS experiment has made a set of measurements that form an emerging picture of the hot dense matter created in a heavy ion collision. These measurements include bulk properties of the system - charged particle multiplicity [1] and extensive measurements of the azimuthal particle distributions and correlations [2] - as well as hard probes such as photons [3], W [4] and Z bosons[5], high pT charged tracks [6], single [7] and di-jet measurements [8], and muons from heavy flavor decays [9].

The ATLAS detector [10] at the LHC covers nearly the entire solid angle around the collision point. It consists of an inner tracking detector surrounded by a thin superconducting solenoid, electromagnetic and hadronic calorimeters, and a muon spectrometer incorporating three superconducting toroid magnet systems. The Inner-detector system (ID) is immersed in a $2 \mathrm{~T}$ axial magnetic field and provides charged particle tracking in the range $|\eta|<2.5$. The high-granularity silicon pixel detector covers the vertex region and is surrounded by the silicon microstrip tracker and transition radiation tracker. The calorimeter system covers the range $|\eta|<4.9$. Within the region $|\eta|<3$.2, electromagnetic calorimetry is provided by barrel and endcap high-granularity lead liquid-argon (LAr) calorimeters, with an additional thin LAr presampler covering $|\eta|<1.8$. Forward calorimeters (FCal) are located in the range $3.1<|\eta|<4$.9. The muon spectrometer comprises separate trigger and high-precision tracking chambers that measure the deflection of muons in a magnetic field generated by superconducting air-core toroids. The precision chamber system covers the region $|\eta|<2.7$ with trigger coverage in the range $|\eta|<2.4$.

\footnotetext{
a e-mail: zvi.citron@weizmann.ac.il
} 


\section{Bulk Properties}

In the new energy regime available for heavy ion collisions at the LHC, the charged particle multiplicity as a function of centrality may be studied to learn about the initial entropy production in the created hot dense matter. Figure 1 shows $\mathrm{dN}_{c h} / \mathrm{d} \eta /\left(N_{\text {part }} / 2\right)$ for several measurements as a function of $\sqrt{s_{N N}}$ (top) and the multiplicity as a function of $N_{\text {part }}$ [1]. Several striking characteristics are observable in the figure: it is clear that the multiplicity in central $A+A$ collisions grows faster than in $p+p$, and that log scaling, which could plausibly describe the data up to RHIC energies, is ruled out by the LHC data (see [11],[12], and [1] as well as references therein for a discussion of the scaling models). A consistent scaling description of the data from low to high energies is not yet clear.

Looking at the centrality distribution, it is noteworthy that the shape of the $N_{\text {part }}$ dependence is consistent at both RHIC and LHC energies, and the absolute magnitude is different by a factor approximately 2.15 independent of centrality.

\section{Particle Flow}

An important observable used to understand the hot dense medium is the azimuthal anisotropy of particle emission. At low $p_{\mathrm{T}}(\lesssim 4 \mathrm{GeV})$, this anisotropy results from a pressure-driven anisotropic expansion of the created matter, with more particles emitted in the direction of the largest pressure gradients. The observed azimuthal anisotropy is customarily expressed as a Fourier series in azimuthal angle $\phi$ :

$$
d N / d \phi=N\left(1+2 \sum_{n} v_{n} \cos \left(n\left(\phi-\Phi_{n}\right)\right)\right)
$$

where $\Phi_{n}$ is the $n$-th order reaction plane and $v_{n}$ are the amplitudes of the Fourier expansion. In typical non-central heavy ion collisions where the nuclear overlap region has an "elliptic" shape (or quadrupole asymmetry) on average, the azimuthal anisotropy is expected to be dominated by the $\mathrm{v}_{2}$ component. However, it was recently pointed out that the positions of the nucleons in the overlap region can fluctuate to create matter distributions with additional shape components, such as dipole $(\mathrm{n}=1)$, triangular $(\mathrm{n}=3)$, and higher asymmetries. As shown in Figure 2 considering all the Fourier components up to $n=6$, allows a precise reconstruction of the two particle $\Delta \phi$ correlations [2]. This implies that the shape observed in two particle correlations at low $p_{\mathrm{T}}$ can be largely attributed to geometric and collective phenomena and does not involve jet quenching.

\section{Electroweak Bosons}

In order to understand the energy loss of of color-charge carriers in the medium, a baseline measurement of the production rates of electroweak bosons that do not interact via the strong force is made. Measurements of both photons [3] and $Z$ bosons $(Z \rightarrow e e$ and $Z \rightarrow \mu \mu)$ [5] have been made using the $2011 \mathrm{~Pb}+\mathrm{Pb}$ dataset and the particle yields scaled by the appropriate $\left\langle N_{\text {coll }}\right\rangle$ compared in different centrality bins as shown in Figure 3. As seen in the figure, the particle production of photons and $\mathrm{Z}$ bosons scales with $\left\langle N_{\text {coll }}\right\rangle$ confirming that within the experimental precision electroweak bosons are not effected by the medium, and that the binary collision model is correct for non-color charge carrying particles.

\section{Color Sensitive Probes}

Unlike the color-neutral electroweak bosons color-charge carrying particles show a marked break from $\left\langle N_{\text {coll }}\right\rangle$ scaling. Inclusive single particle measurements as well as two particle correlations have 

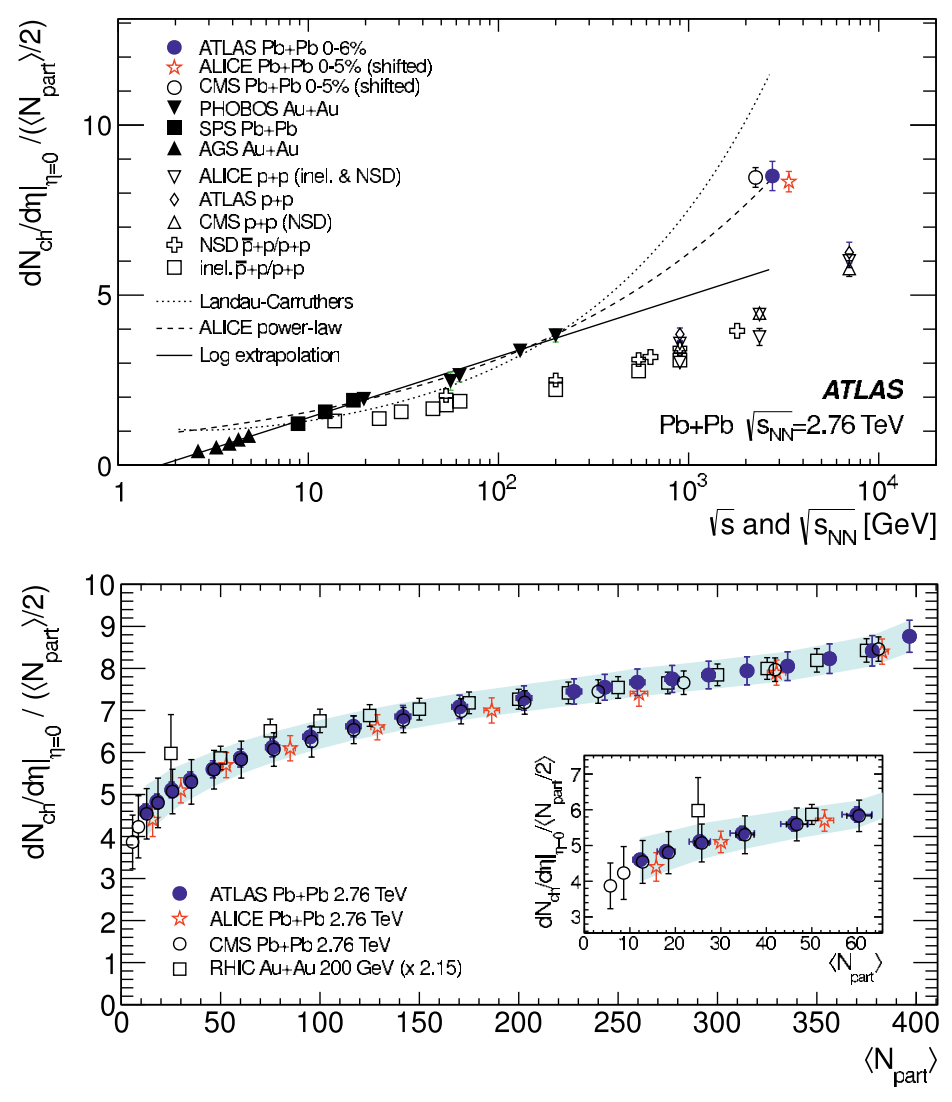

Figure 1. Top: $\sqrt{s_{N N}}$ dependence of the charged particle density per colliding nucleon pair $\mathrm{dN} c h / \mathrm{d} \eta /\left(N_{\text {part }} / 2\right)$ from a variety of measurements in $\mathrm{dN}_{c h} / \mathrm{d} \eta(\eta=0)$ in $\mathrm{p}+\mathrm{p}$ and $\mathrm{p}+\overline{\mathrm{p}}$ collisions (inelastic and non-single diffractive results) and from central A+A collisions, including the ATLAS 0-6\% centrality measurement reported here for $|\eta|<0.5$ and the previous 0-5\% centrality ALICE and CMS measurements (points shifted horizontally for clarity). The curves show different expectations for the $\sqrt{s_{N N}}$ dependence: results of a Landau hydrodynamics calculation (dotted line), a $\sqrt{s_{N N}}$ extrapolation of RHIC and SPS data proposed by ALICE (dashed line), a logarithmic extrapolation of RHIC and SPS data (solid line). Bottom: $\mathrm{dN}_{c h} / \mathrm{d} \eta /\left(N_{\text {part }} / 2\right)$ vs $N_{\text {part }}$ for $2 \%$ centrality intervals over $0-20 \%$ and $5 \%$ centrality intervals over $20-80 \%$. Error bars represent combined statistical and systematic uncertainties on the $\mathrm{dN}_{c h} / \mathrm{d} \eta(\eta=0)$ measurements, whereas the shaded band indicates the total systematic uncertainty including $N_{\text {part }}$ uncertainties. The RHIC measurements have been multiplied by 2.15 to allow comparison with the $\sqrt{s_{N N}}=2.76 \mathrm{TeV}$ results. The inset shows the $N_{\text {part }}<60$ region in more detail. Figure is from reference [1].

suggested that jets are being "quenched" in the medium, and much effort has been made to understand the mechanisms of this quenching. The suppression of particle production may be quantified using the nuclear modification factor, $R_{\mathrm{CP}}$ :

$$
R_{\mathrm{CP}}=\frac{\left\langle N_{\mathrm{coll}}\right\rangle(P)}{\left\langle N_{\mathrm{coll}}\right\rangle(C)} \frac{\left(1 / N_{e v t, C}\right) d^{2} N_{C} / d \eta d p_{\mathrm{T}}}{\left(1 / N_{e v t, P}\right) d^{2} N_{P} / d \eta d p_{\mathrm{T}}}
$$



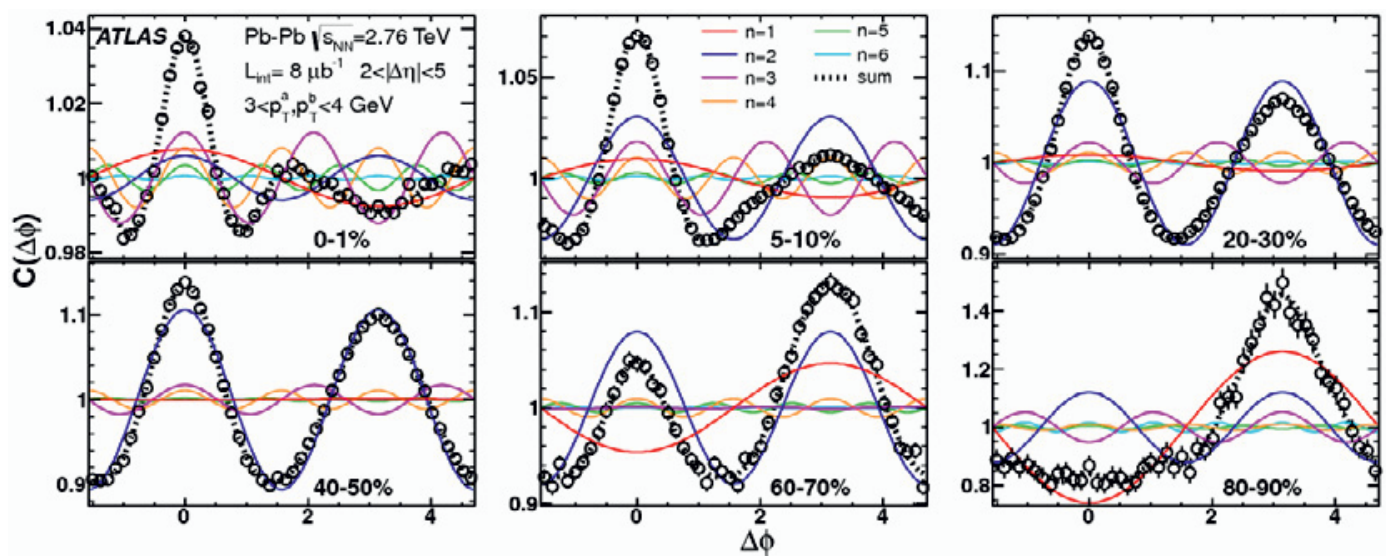

Figure 2. Centrality dependence of $\Delta \phi$ correlations for $3<p_{T}^{a}$, $\mathrm{p}_{T}^{b}<4 \mathrm{GeV}$. A rapidity gap of $2<|\Delta \eta|<$ 5 is required to isolate the long-range structures of the correlation functions, i.e. the near-side peaks reflect the "ridge" instead of the autocorrelations from jet fragments. The error bars on the data points indicate the statistical uncertainty. The superimposed solid lines (thick-dashed lines) indicate contributions from individual $\mathrm{v}_{n, n}$ components (sum of the first six components). Figure is from reference [2].

where $\mathrm{C}$ and $\mathrm{P}$ refer to central peripheral event classes, respectively. Figure 4 shows the $R_{\mathrm{CP}}$ of inclusive charged particles measured by ATLAS [6], and as expected from previous results displays a clear suppression of particles in central $\mathrm{Pb}+\mathrm{Pb}$ events. The nuclear modification factor drops to a minimum value comparable to the measurements made at RHIC energies [13] at $p_{\mathrm{T}} \approx 7 \mathrm{GeV}$, before rising to approximately $50 \%$ at $p_{\mathrm{T}}=30 \mathrm{GeV}$. No psuedorapidity dependence is observed.

To go beyond the inclusive particle measurement and look more closely at the jets themselves, ATLAS has made the first direct observation of jet quenching by measuring the imbalance of di-jet energies [8]. The imbalance is expressed in terms of the asymmetry, $A_{J}$, of two azimuthally correlated jets:

$$
A_{J}=\frac{E_{T 1}-E_{T 2}}{E_{T 1}+E_{T 2}}, \quad \Delta \phi>\frac{\pi}{2}
$$

Figure 5 shows the asymmetry and $\Delta \phi$ distributions for $\mathrm{Pb}+\mathrm{Pb}, \mathrm{p}+\mathrm{p}$, and simulated events. In the more peripheral $\mathrm{Pb}+\mathrm{Pb}$ events as in $\mathrm{p}+\mathrm{p}$ and simulation, $A_{J}$ is peaked at zero implying no relative modification of the jet energy, i.e. no quenching. However in more central $\mathrm{Pb}+\mathrm{Pb}$ events $A_{J}$ this is no longer the case, showing that there is a relative quenching of one of the two jets due to the presence of the dense medium. Despite this quenching of the jet energy, $\Delta \phi$ distributions remain consistent even in the most central collisions. In addition to the jet energy imbalance one may measure the nuclear modification factor, $R_{\mathrm{CP}}$, of the fully reconstructed jets rather than inclusive single particles [7]. Figure 6 shows the $R_{\mathrm{CP}}$ of jets as a function of $N_{\text {part }}$, clearly demonstrating the suppression of the overall jet yield in addition to the imbalance seen in Figure 5. The suppression observed is independent of the jet $p_{\mathrm{T}}$ within the experimental uncertainties. In addition to jets reconstructed using the anti- $\mathrm{k}_{t}$ algorithm with $\mathrm{R}=0.4$, jets were reconstructed using $\mathrm{R}=0.2,0.3$, and 0.5 . This allows the observation of a slight but significant increase in the magnitude of the suppression with smaller $\mathrm{R}$ at low $p_{\mathrm{T}}$.

As a further look into the behavior of color charged objects in the medium, the nuclear modification factor of muons from heavy flavor decay is formed. A template fitting method is used to estimate 

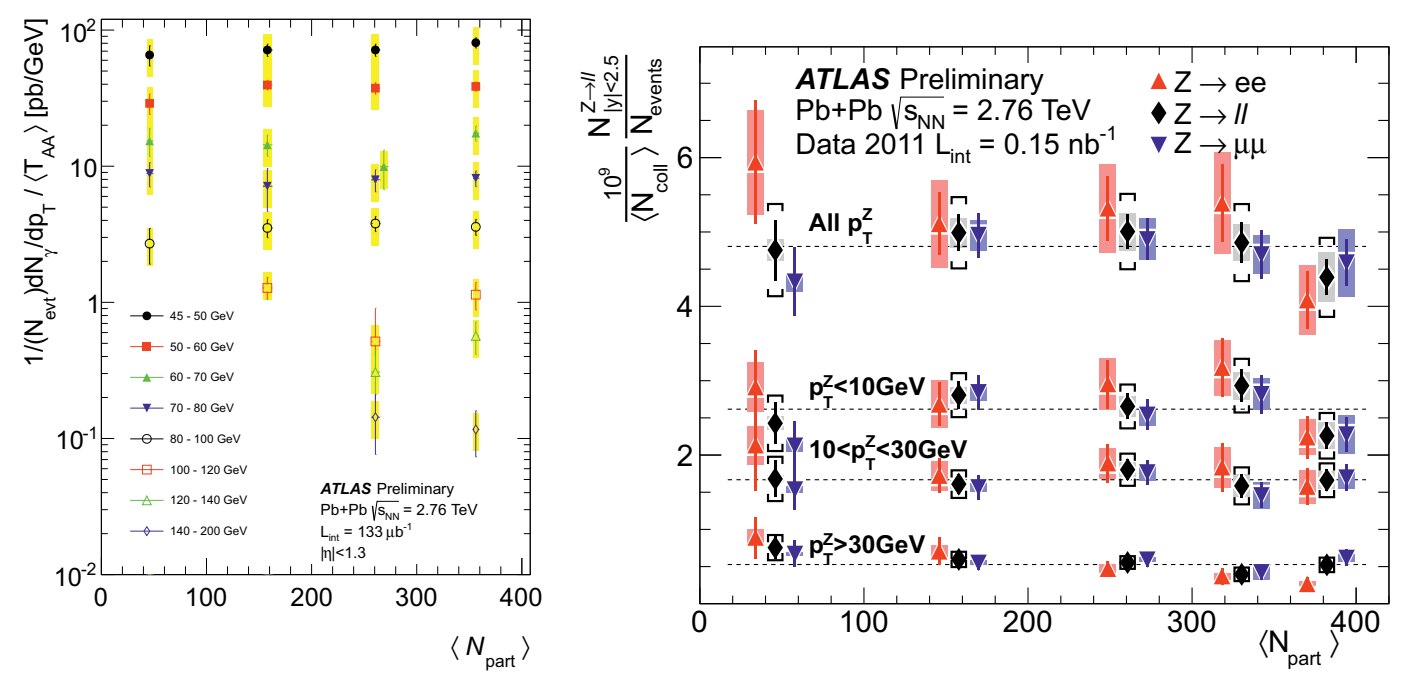

Figure 3. Left: Centrality dependence of the photon yield per event in several $p_{\mathrm{T}}$ bins, scaled by the average nuclear thickness function $\left\langle T_{\mathrm{AA}}\right\rangle$ (equivalent to $\left\langle N_{\text {coll }}\right\rangle$ divided by the total inelastic $\mathrm{p}+\mathrm{p}$ cross section) for that centrality interval. The horizontal axis is the average number of participants $\left\langle N_{\text {part }}\right\rangle$ for each selected centrality interval. Statistical errors are shown by the error bars. Systematic uncertainties on the photon yields are shown by the yellow bands. Figure is from reference [3]. Right: Centrality dependence of $Z$ boson yields divided by $\left\langle N_{\text {coll }}\right\rangle$, measured in $\left|y_{Z}\right|<2.5$. Results for $Z \rightarrow e e$ (upward pointing triangles) and $Z \rightarrow \mu \mu$ (downward pointing triangles) channels are shifted left and right respectively (for visibility) from their weighted average (diamonds) which is plotted at the nominal $N_{\text {part }}$ value. The statistical (bars) and systematic (shaded bands) uncertainties are calculated using the appropriately weighted average of the two contributing sources. Brackets show the combined uncertainty including the uncertainty on $\left\langle N_{\text {coll }}\right\rangle$. The dashed lines are constant fits to the combined results. Figure is from reference [5].

the portion of muons coming from heavy flavor decays and their $R_{\mathrm{PC}}\left(R_{\mathrm{PC}}=1 / R_{\mathrm{CP}}\right.$ used in order to minimize the impact of fluctuations in the low statistics peripheral event sample) is shown in Figure 7. The figure shows that although the particle yield is suppressed in central events compared to peripheral events, the shape of the modification in $p_{\mathrm{T}}$ is different from that shown in Figure 4 for inclusive charged particles. 
EPJ Web of Conferences
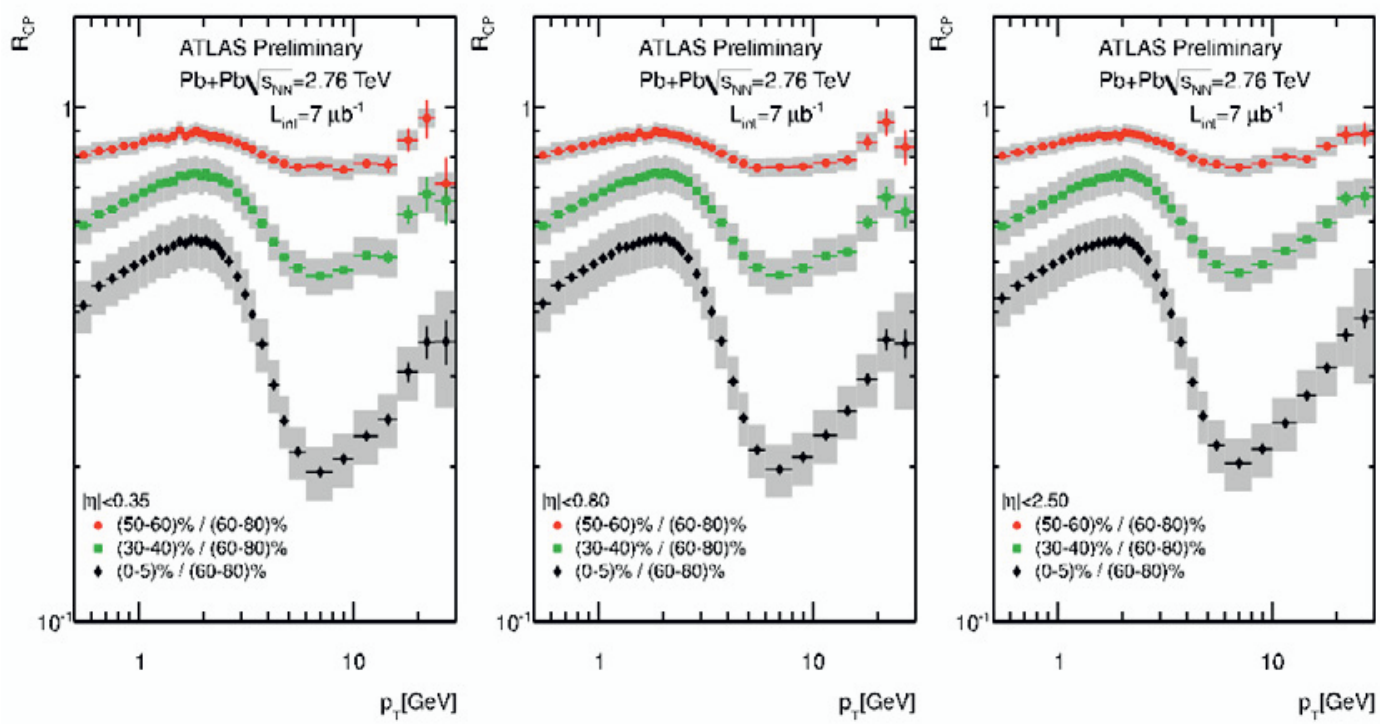

Figure 4. $R_{\mathrm{CP}}$ extracted from the inclusive charged particle distributions in three different $\eta$ ranges, and three centrality combinations: with $0-5 \%, 30-40 \%$ and $50-60 \%$ as numerators and a common peripheral sample (60$80 \%$ ) as denominator. Statistical errors are shown with vertical lines and the overall systematic uncertainty at each point is shown with gray boxes. Figure is from reference [6]. 

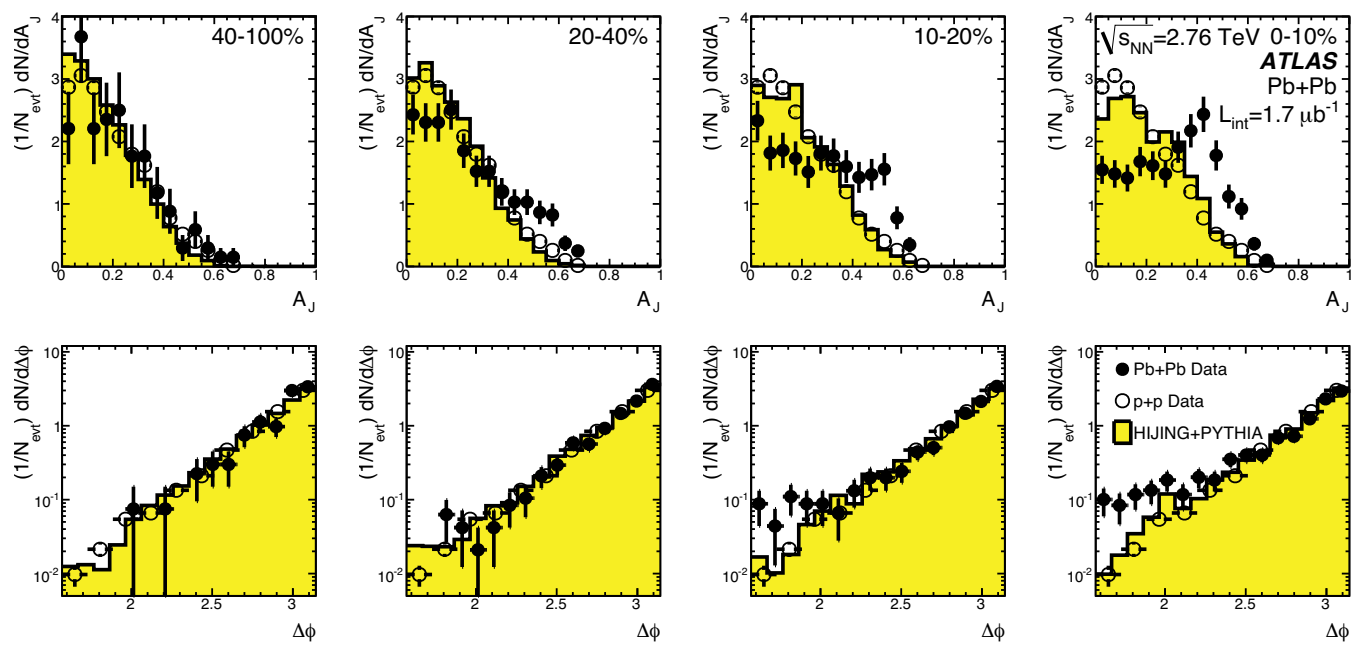

Figure 5. Top: Di-jet asymmetry distributions for data (points) and unquenched HIJING with superimposed PYTHIA dijets (solid yellow histograms), as a function of collision centrality (left to right from peripheral to central events). Proton-proton data from $\sqrt{s_{N N}}=7 \mathrm{TeV}$, analyzed with the same jet selection, is shown as open circles. Bottom: Distribution of $\Delta \phi$, the azimuthal angle between the two jets, for data and HIJING+PYTHIA, also as a function of centrality. Figure is from reference [8].

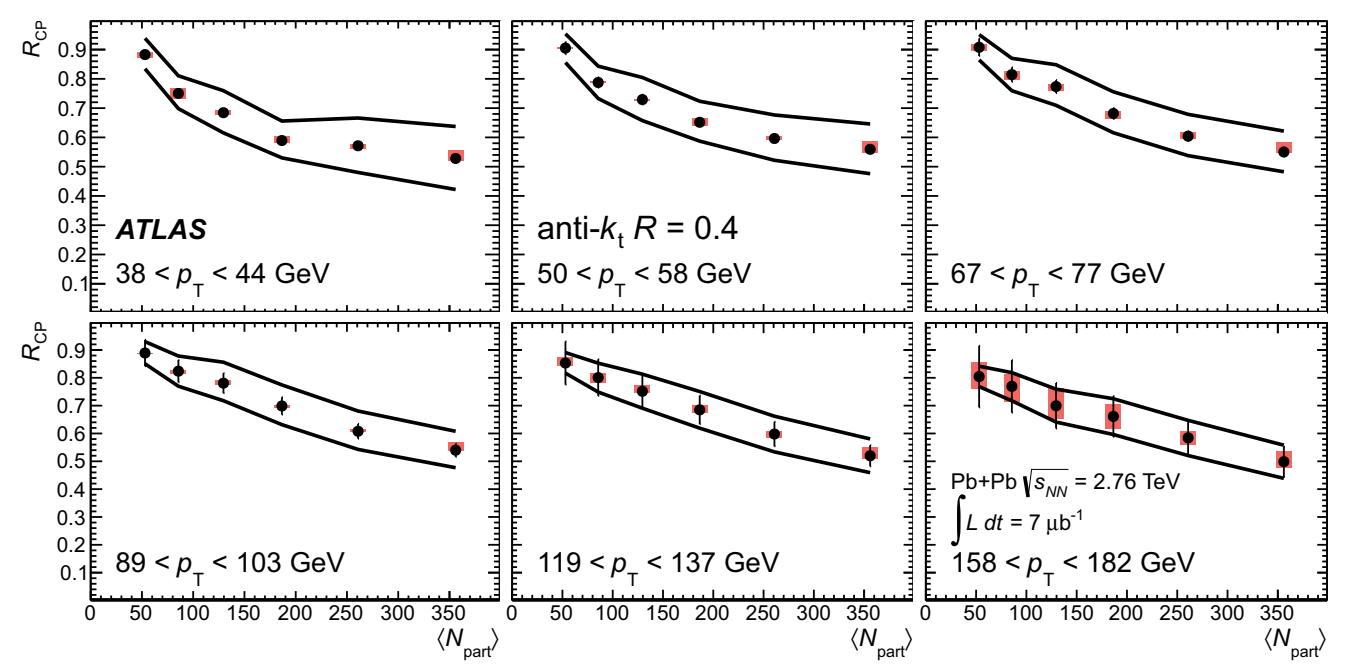

Figure 6. Unfolded $R_{\mathrm{CP}}$ values as a function of $N_{\text {part }}$ for $\mathrm{R}=0.4$ anti- $\mathrm{k}_{t}$ jets in six $p_{\mathrm{T}}$ bins. The error bars indicate statistical errors from the unfolding; the shaded boxes indicate point-to-point systematic errors that are only partially correlated. The solid lines indicate systematic errors that are fully correlated between all points. The horizontal errors indicate systematic uncertainties on $N_{\text {part }}$. Figure is from reference [7]. 
EPJ Web of Conferences

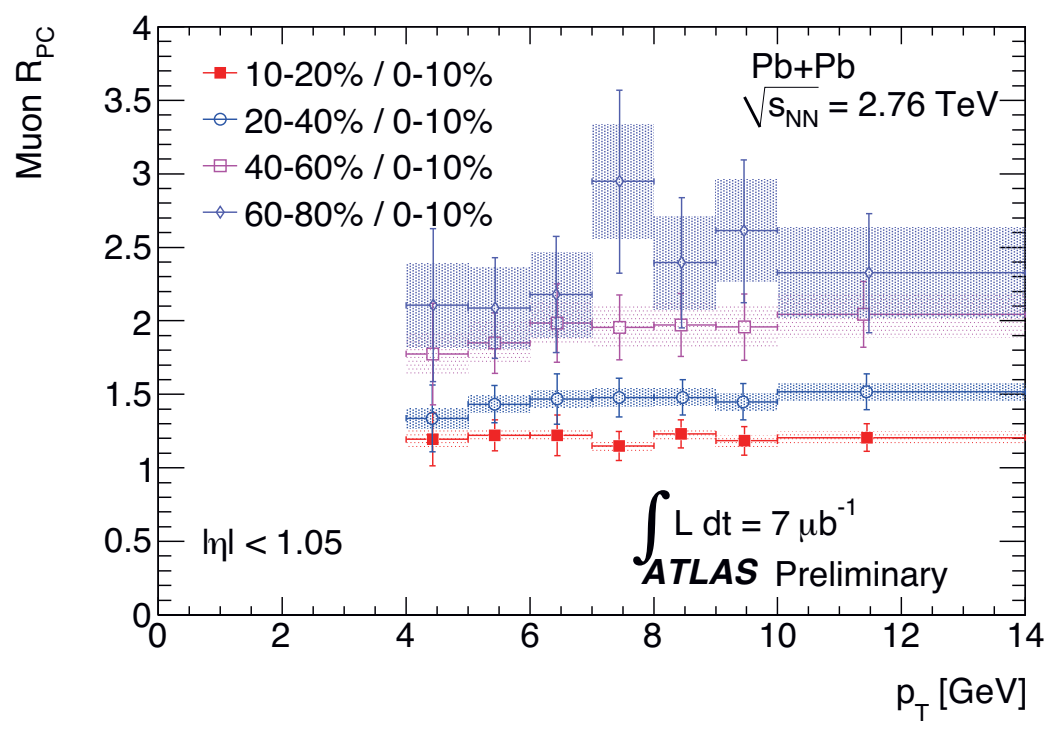

Figure 7. Muons from heavy flavor decay peripheral to central ratio, $R_{\mathrm{PC}}$, as a function of $p_{\mathrm{T}}$ for different centrality bins. The points are shown at the mean transverse momentum of the muons in the given $p_{\mathrm{T}}$ bin. The error bars include both statistical and systematic uncertainties. The contribution of the systematic uncertainties from $\left\langle N_{\text {coll }}\right\rangle$ and efficiency, which are fully correlated between $p_{\mathrm{T}}$ bins, are indicated by the shaded boxes. Figure is from reference [9]. 


\section{Summary}

The ATLAS heavy-ion program has had two successful years of data taking. Among the measurements made, the charged particle multiplicity has shown $\log \sqrt{s_{N N}}$ scaling to be broken, while the centrality shape remains consistent with lower energy. The measurement of the azimuthal distribution of produced particles has shown that initial geometry largely explains correlation function structure, and has greatly increased our knowledge of the medium's early geometry and its subsequent expansion.

In addition the measurement of electro-weak bosons, photons and $\mathrm{Z}$ bosons, which do not strongly interact sets a baseline for hard probes that are not effected by the hot and dense medium. The $\left\langle N_{\text {coll }}\right\rangle$ scaling behavior of electro-weak bosons demonstrates that the Glauber binary collision model describes well the collision geometry and the incidence of hard scatterings in the event. Further, these measurements lay the groundwork for the use of photons and $\mathrm{Z}$ bosons as a calibration for modified jets in boson + jet events.

This baseline of unmodified color neutral objects is complemented by the studies of color charge sensitive objects, among them inclusive charged particles, jets, and muons from heavy flavor decays, whose modification is studied in order to learn about the properties of the medium. The nuclear modification factor of inclusive charged particles shows suppression similar to that seen at RHIC energies at low momentum, but also clearly shows an increase that at higher $p_{\mathrm{T}}$ that was not clear in the RHIC data. This momentum dependence is in contrast to the strikingly flat shape in $p_{\mathrm{T}}$ of the jet nuclear modification factor and the somewhat different suppression seen in muons from heavy flavor decay. The precise mechanisms of the energy quenching due to the medium are not yet clear, however these measurements are shedding significant light on them.

This research is supported by FP7-PEOPLE-IRG (grant 710398), Minerva Foundation (grant 7105690) and by the Israel Science Foundation (grant 710743).

\section{References}

[1] ATLAS Collaboration, Phys. Lett. B710, (2012) 363-382

[2] ATLAS Collaboration, Phys. Rev. C86, 014907 (2012)

[3] ATLAS Collaboration, ATLAS-CONF-2012-051 (2012) https://cdsweb.cern.ch/record/1451913

[4] ATLAS Collaboration, Phys. Lett. B697, (2011) 294-312

[5] ATLAS Collaboration, ATLAS-CONF-2012-052 (2012) https://cdsweb.cern.ch/record/1451930

[6] ATLAS Collaboration, ATLAS-CONF-2011-079 (2011) https://cdsweb.cern.ch/record/1355702

[7] ATLAS Collaboration, arXiv:1208.1967, (2012) submitted to Phys. Lett. B

[8] ATLAS Collaboration, Phys. Rev. Lett. 105, (2010) 252303

[9] ATLAS Collaboration, ATLAS-CONF-2012-050 (2012) https://cdsweb.cern.ch/record/1451883

[10] ATLAS Collaboration, Journal of Instrumentation 3, (2008) no. 08, S08003

[11] PHOBOS Collaboration, Phys.Rev.C83, 024913 (2011)

[12] ALICE Collaboration, Phys. Rev. Lett. 105, 252301 (2010)

[13] PHENIX Collaboration, Phys. Rev. C 69 (2004) 034910 